\title{
Isoforms of the neuronal glutamate transporter gene, SLC1A1/EAAC1, negatively modulate glutamate uptake: relevance to obsessive-compulsive disorder
}

\author{
B Porton ${ }^{1,2}$, BD Greenberg ${ }^{1,2}$, K Askland $^{1,2}$, LM Serra ${ }^{1}$, J Gesmonde $^{3}$, G Rudnick ${ }^{3}$, SA Rasmussen ${ }^{1,2}$ and H-T Kao ${ }^{1,2}$
}

The SLC1A1 gene, which encodes the neuronal glutamate transporter, EAAC1, has consistently been implicated in obsessivecompulsive disorder $(O C D)$ in genetic studies. Moreover, neuroimaging, biochemical and clinical studies support a role for glutamatergic dysfunction in OCD. Although SLC1A1 is an excellent candidate gene for OCD, little is known about its regulation at the genomic level. Here, we report the identification and characterization of three alternative SLC1A1/EAAC1 mRNAs: a transcript derived from an internal promoter, termed $\mathrm{P} 2$ to distinguish it from the transcript generated by the primary promoter (P1), and two alternatively spliced mRNAs: ex2skip, which is missing exon 2, and ex11skip, which is missing exon 11. All isoforms inhibit glutamate uptake from the full-length EAAC1 transporter. Ex2skip and ex11skip also display partial colocalization and interact with the full-length EAAC1 protein. The three isoforms are evolutionarily conserved between human and mouse, and are expressed in brain, kidney and lymphocytes under nonpathological conditions, suggesting that the isoforms are physiological regulators of EAAC1. Moreover, under specific conditions, all SLC1A1 transcripts were differentially expressed in lymphocytes derived from subjects with OCD compared with controls. These initial results reveal the complexity of SLC1A1 regulation and the potential clinical utility of profiling glutamatergic gene expression in OCD and other psychiatric disorders. Translational Psychiatry (2013) 3, e259; doi:10.1038/tp.2013.35; published online 21 May 2013

\section{Introduction}

Multiple lines of evidence suggest a role for glutamatergic dysfunction in obsessive-compulsive disorder (OCD). Glutamate levels are abnormal in the brains of individuals with OCD, ${ }^{1-5}$ and are increased in the cerebrospinal fluid of individuals with OCD. ${ }^{6,7}$ Studies in mice with OCD-like behaviors also suggest abnormal glutamatergic neurocircuitry in the striatal region. ${ }^{8-10}$ Open label studies and case reports suggest that glutamatergic agents, such as riluzole, ${ }^{11-13}$ memantine,${ }^{14-18}$ and $\mathrm{N}$-acetylcysteine,${ }^{19}$ might have therapeutic effects in OCD. Importantly, several independent genetic studies consistently implicate the SLC1A1 gene, which encodes the neuronal glutamate transporter, EAAC1 (also known as EAAT3), as a candidate gene for OCD. ${ }^{20-27}$ Despite the strength of these findings, our knowledge of how SLC1A1 gene expression is modulated via transcription and alternative splicing remain unclear.

Here, using a bioinformatics approach, we have identified and characterized three SLC1A1 alternative isoforms: a transcript derived from an internal promoter (P2) and two alternatively spliced transcripts, ex2skip (missing exon 2) and ex11skip (missing exon 11). These isoforms act as negative modulators of SLC1A1/EAAC1 function, and inhibit glutamate transport via the EAAC1 transporter. Furthermore, all SLC1A1 transcripts were differentially regulated in OCD compared with controls in a peripheral lymphocyte system for analyzing neural gene transcription. These findings have relevance for understanding glutamate biology in OCD and other psychiatric disorders.

\section{Materials and methods}

\section{Molecular biology}

Bioinformatics. Internet resources were used to obtain human and mouse EAAC1 gene sequences (http://www. ncbi.nlm.nih.gov/) and exon organization (using the University of California Santa Cruz genome browser at http://genome.ucsc.edu/). Relevant genomic sequences were scanned for potential promoters using the programs Promoter 2.0 (http://www.cbs.dtu.dk/services/Promoter/) and CpGPlot (http://www.ebi.ac.uk/Tools/emboss/cpgplot/index. $\mathrm{html}$ ) to detect $\mathrm{CpG}$ islands. ${ }^{28}$ Human and mouse expressed sequence tags that were identical to EAAC1 were downloaded from Genbank or directly from the UCSC genome browser site. Two previous publications also suggest the existence of alternative transcripts derived from the SLC1A1/ EAAC1 locus. ${ }^{29,30}$

Cloning of SLC1A1/EAAC1 alternative transcripts. Relevant sequences were amplified by the PCR from complementary DNA derived from HEK-293 cell RNA. Standard recombinant DNA technology ${ }^{31}$ was used to assemble each EAAC1

\footnotetext{
${ }^{1}$ Department of Psychiatry and Human Behavior, Brown University, BioMedical Center, Providence, RI, USA; ${ }^{2}$ Butler Hospital, Providence, RI, USA and ${ }^{3}$ Department of Pharmacology, Yale University School of Medicine, New Haven, CT, USA

Correspondence: Dr H-T Kao, Department of Psychiatry and Human Behavior, Brown University, BioMedical Center, 171 Meeting Street, Room 187, Providence, RI 02912, USA.

E-mail: hung-teh_kao@brown.edu

Keywords: EAAC1; EAAT3; internal promoter; obsessive-compulsive disorder; protein isoform; SLC1A1

Received 19 November 2012; revised 10 February 2013; accepted 8 April 2013
} 
isoform into the vector pcDNA3.1 (Invitrogen Life Technologies, Grand Island, NY, USA) and engineered with a carboxyl terminal FLAG or hemagglutinin (HA) tag (HA-tag). ${ }^{32}$ All assembled DNAs were confirmed by sequencing.

Quantitative PCR (qPCR). RNA was extracted from tissues using Trizol reagent (Invitrogen Life Technologies) and complementary DNA was generated in the presence of random primers. qPCR was carried out using the Platinum SybrGreen qPCR SuperMix-UDG with ROX (Invitrogen Life Technologies) in the presence of $0.4 \mu \mathrm{m}$ primers (Supplementary Table 1). Data were collected using the ABI HT7900 Fast Sequence Detection System (Applied Biosystems, Grand Island, NY, USA). Data were analyzed as previously described, ${ }^{33}$ taking into account amplification efficiency $^{34}$ and normalization to a housekeeping control ( $\beta 2$-microglobulin for human EAAC1 and $\beta$-actin for mouse EAAC1).

\section{Biological materials}

Human brain samples. Frozen sections from human frontal lobe and striatum were obtained from the Stanley Foundation Neuropathology Consortium.

Mouse brain and neurons. Brains were obtained from adult C57/BL6 mice (Jackson Laboratory, Bar Harbor, ME, USA). Neuronal cultures from neonatal C57/BL6 cortex were prepared using previously published methods. ${ }^{35}$ Experimental protocols were approved by the Institutional Animal Care and Use Committee at Brown University.

Human lymphocytes. Adult subjects for the OCD group: (a) were between 18 and 65 years of age, (b) met DSM-IV criteria for OCD, (c) had Yale-Brown obsessive-compulsive scale scores $>16$. Control subjects: (a) were between 18 and 65 years of age, (b) did not meet DSM-IV criteria for OCD. Participants were excluded if they (a) met current DSM-IV criteria for alcohol or drug abuse/dependence (except nicotine) within the past 6 months, (b) met current DSM-IV criteria for anorexia or bulimia nervosa, (c) met lifetime DSM-IV criteria for a psychotic disorder or bipolar disorder, (d) were currently suicidal or homicidal, (e) had marked organic impairment.

Participants were recruited from September 2009 to October 2010 through clinician referrals from an OCD Research Center at Butler Hospital, a private psychiatric hospital in Providence, Rhode Island, USA. Permission from the hospital's Institutional Review Board was obtained to conduct this pilot study. The OCD group consisted of eight females and six males and the control group had four females and five males. There was no significant difference in gender between the two groups, $\chi^{2}(3, N=23)=0.56, P=0.59$. The mean age of the OCD group $\left(M_{\text {ocd }}=40.00\right.$, s.d. $\left.=14.16\right)$ did not differ significantly from that of controls $\left(M_{\mathrm{c}}=37.17\right.$, s.d. $=13.93 ; t(21)=0.49, P=.63)$. There was no significant difference in the ages of males in the OCD group $\left(M_{\text {ocd }}=32.17\right.$, s.d. $\left.=12.38\right)$ compared with the control group $\left(M_{\mathrm{c}}=44.20\right.$, s.d. $\left.=14.06 ; t(9)=1.51, P=0.17\right)$. However, the mean age of the females in the OCD group $\left(M_{\text {ocd }}=45.88\right.$, s.d. $=12.32$ ) was significantly older than the mean age of females in the control group $\left(M_{\mathrm{c}}=28.25\right.$, s.d. $=8.10$; $t(10)=2.56, P=0.02)$. Accordingly, all statistical analyses were not segregated by gender.

All subjects with OCD (except for one unmedicated subject) were treated with an selective serotonin re-uptake inhibitor, and some had additional antidepressants or adjuvant medications. None of the OCD subjects were on antipsychotics. None of the controls had a history of OCD and none were treated with psychotropics.

Lymphocytes were purified using a ficoll-hypaque gradient (GE Healthcare Biosciences, Piscataway, Township, NJ, USA) according to the manufacturer's specifications, and cultured in RPMI media with $10 \%$ fetal calf serum, $100 \mathrm{U} \mathrm{ml}^{-1}$ penicillin and $100 \mathrm{~g} \mathrm{ml}^{-1}$ streptomycin in a $5 \% \mathrm{CO}_{2}$ humidified incubator.

Reagents. Dopamine, fluoxetine and trans-retinoic acid were purchased from Sigma-Aldrich (St Louis, MO, USA).

\section{Cell biology}

Cell lines. HEK-293 and HeLa cells were maintained at $37^{\circ} \mathrm{C}$ in a $5 \% \mathrm{CO}_{2}$-humidified incubator in Dulbecco's modified Eagle medium with $10 \%$ fetal calf serum, $2 \mathrm{~mm}$ L-glutamine, $100 \mathrm{U} \mathrm{ml}^{-1}$ penicillin and $100 \mathrm{\mu g} \mathrm{ml}^{-1}$ streptomycin. All transfections were performed with Fugene HD (Promega Corporation, Madison, WI, USA) using DNA diluted in Dulbecco's modified Eagle medium and specifications outlined by the manufacturer.

Glutamate uptake. HEK-293 cells were grown on Isoplate 96well TC plates (Perkin Elmer Life Science Research, Waltham, MA, USA) pretreated with $1 \mathrm{mg} \mathrm{ml}^{-1}$ poly-D-lysine $(>3 \mathrm{~h}$ at $37^{\circ} \mathrm{C}$ ). When cells were approximately $50 \%$ confluent, $60 \mathrm{ng}$ of DNA was transfected per well. Two days post transfection, culture media was removed and cells were incubated in $10 \mu \mathrm{M}\left[{ }^{3} \mathrm{H}\right]$-glutamate (Perkin Elmer-New England Nuclear, Waltham, MA, USA) in phosphate-buffered saline (PBS) supplemented with $2.2 \mathrm{~mm} \mathrm{CaCl}_{2}$ (PBS-Ca). At designated times, glutamate was removed, cells rinsed twice with $\mathrm{PBS}-\mathrm{Ca}$, and lysed in $0.2 \mathrm{M} \mathrm{NaOH}$. A volume of $150 \mu \mathrm{l}$ of Optifluor was then added to each well and counted with the Wallac Microbeta plate counter (Perkin Elmer).

Confocal microscopy. HeLa cells were grown on poly-Dlysine-treated glass coverslips and transfected with various EAAC1 constructs. Two days post transfection, cells were fixed in $4 \%$ paraformaldehyde in PBS, lysed with $0.3 \%$ Triton $\mathrm{X}-100$ and blocked in 10\% donkey serum in Tris-buffered saline with $0.05 \%$ Tween 20 (TBST) overnight at $4{ }^{\circ} \mathrm{C}$. Fixed cells were then incubated overnight with antibodies (diluted in TBST) directed against FLAG-tag (M2 monoclonal antibody, Agilent, Santa Clara, CA, USA) or HA-tag (Santa Cruz Biotechnology, Santa Cruz, CA, USA, HA-probe rabbit antibody $\mathrm{Y}$-11). Coverslips were washed with TBST and the primary antibodies revealed by incubation with Alexa647conjugated anti-mouse antibody and Alexa555-conjugated anti-rabbit antibody (Invitrogen Life Technologies). Cells were visualized using the Zeiss LSM 510 confocal microscope (Zeiss, Thornwood, NY, USA). Images were analyzed using ImageJ and the plug-in for colocalization analyses. ${ }^{36}$ 
Pulldown assays. HeLa cells were transfected with combinations of EAAC1 DNA constructs consisting of HA-tagged P1 and FLAG-tagged P1 or FLAG-tagged isoforms. Two days post transfection, cells were harvested, lysed in mammalian protein extraction reagent (Pierce Chemical Company, Rockford, IL, USA). FLAG-tagged proteins were isolated from lysates using M2 antibody affinity gel (Kodak Scientific Imaging Systems, Rochester, NY, USA), and immunoblotted to an antibody against HA-tag. Immunoblots were visualized using the Odyssey Infrared Imaging System (LI-COR Biosciences, Lincoln, NE, USA).

\section{Results}

Identification of a second promoter (P2) within the SLC1A1 gene. To determine if the SLC1A1 gene is subject to regulation by more than one promoter, we analyzed both human and mouse genes using publicly available DNA sequences (Figure 1). The structures of both the human and mouse SLC1A1 gene are very similar: both are encoded by 12 exons, the boundaries of each exon are virtually identical, and the distribution of exons along the genome is very similar (Figures 1a and b). Promoter prediction programs (Promoter
2.0 and CpGPlot) revealed a single CpG island in exon 1, representing the primary promoter for SLC1A1, and a novel potential internal promoter in both humans and mice upstream of exon 5 (Figures $1 \mathrm{a}$ and b). Transcription from this site would involve an additional exon upstream of exon 5 (termed X), facilitating the design of primers to specifically detect this transcript, should it exist (Figure 1c). The existence of a second promoter in the mouse SLC1A1 gene has been reported previously, ${ }^{29}$ however, an internal human promoter, which is described here, has not been reported before.

To determine if the predicted second internal promoter is functional, we used human HEK-293 cells, a cell line derived from human embryonic kidney, which is known to express low levels of EAAC1. Indeed, PCR confirmed that the second promoter is capable of driving the expression of a transcript distinct from that derived from the primary promoter (Supplementary Figure 1). We have termed the internal promoter, P2, to distinguish it from the primary promoter, P1 (Figures 1a and b).

Identification of two alternatively spliced SLC1A1/ EAAC1 mRNAs. We also searched for alternatively spliced SLC1A1/EAAC1 mRNAs in human and mouse. In a previous

a

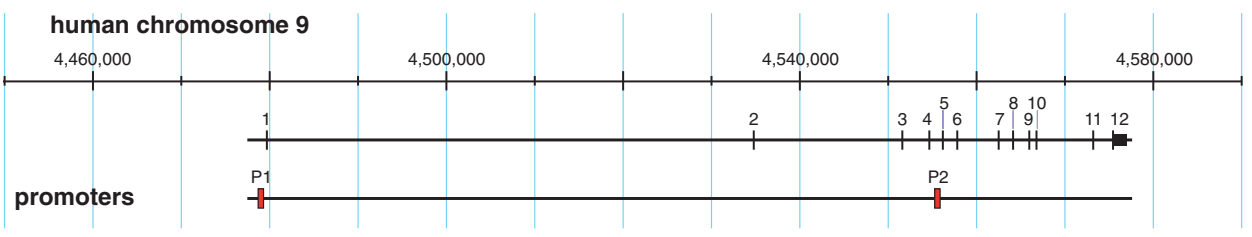

b

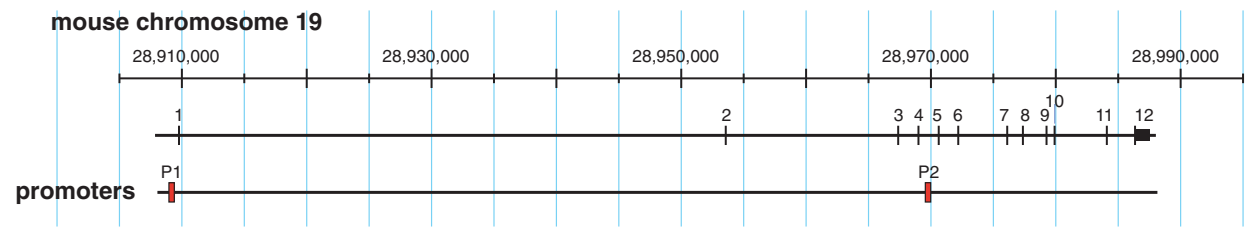

C

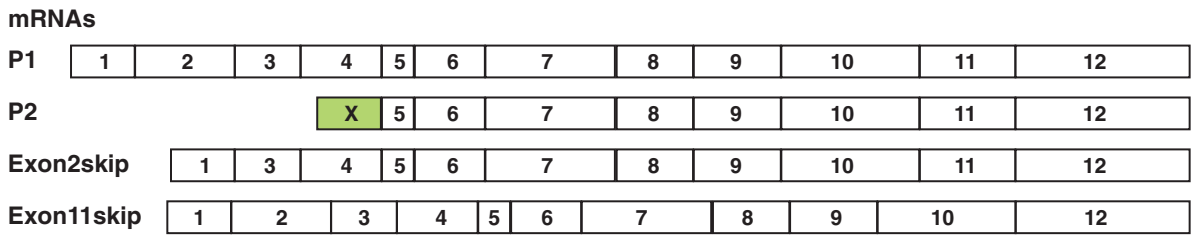

d protein structure

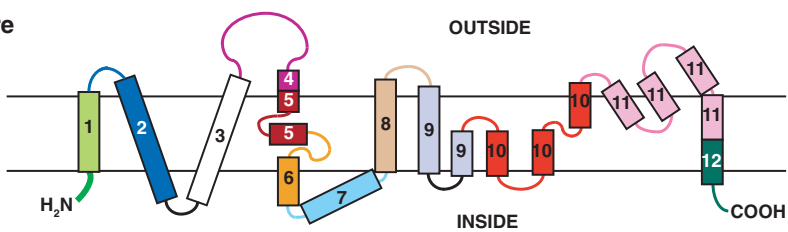

Figure 1 Organization of the human and mouse SLC1A1/EAAC1 genes. (a, b) Linear representation of genes with base pair numbering indicated at the top. The human SLC1A1/EAAC1 gene consists of 12 exons arranged along chromosome 9. There are two functional promoters (designated P1 and P2). The mouse SLC1A1/EAAC1 gene (on chromosome 19) is remarkably similar to the human gene; 11 of its 12 exons are exactly the same size as in humans. The mouse EAAC1 gene also contains two functional promoters, designated P1 and P2. The mouse EAAC1 mRNA is overall $85 \%$ identical to the human sequence and the mouse protein $90 \%$ identical to the human. (c) Predicted exon structure of $\mathrm{mRNA}$ transcripts derived from promoters P1 and P2. (d) Contribution of exons to the protein of EAAC1. Schematic diagram of a single subunit of the EAAC1 protein inserted into the cellular membrane, with the inside and outside of the cell indicated. The diagram is based on alignment of EAAC1 to the glutamate homolog of Pyrococcus horikoshii, whose structure was determined by crystallography. ${ }^{39}$ Number and color indicate the region of the protein encoded by specific exons. 
report, ${ }^{30}$ a truncated human EAAC1 mRNA lacking exon 2 was reported, but a corresponding mouse mRNA was not detected and no functional characterization was carried out. PCR was used to verify that this mRNA is expressed in HEK293 cells (Supplementary Figure 1 ). We termed this mRNA ex2skip (Figure 1).

In addition, we examined human and mouse expressed sequence tags using publicly available data (http:// www.ncbi.nlm.nih.gov). In humans, one expressed sequence tag was identified in which exon 11 was deleted. The potential transcript was termed ex11skip, and has not been described previously. The expression of this mRNA was verified in HEK-293 cells using PCR (Supplementary Figure 1). In mice, no additional transcripts were identified.

Alternative mRNAs in HEK-293 cells were also screened using PCR spanning the entire length of the EAAC1 mRNA, but no other mRNAs were detected.

\section{Abundance of alternative SLC1A1/EAAC1 mRNA tran- scripts in human and mouse. qPCR was used to quantitate the relative abundance of the P2, ex2skip and ex11skip mRNAs in a variety of human and mouse postmortem brain tissues. We utilized the additional exon sequence upstream of exon 5 (exon $X$ ), as well as the unique exon-exon junctions in the alternatively spliced transcripts to design primers for specific amplification of these transcripts. \\ We observed substantial levels of mRNA corresponding to P2 transcription as well as ex2skip and ex11skip relative to the P1 primary transcript in both humans and mice (Figure 2). In humans, the alternatively spliced ex2skip and ex11skip mRNAs were particularly abundant in the striatum, where abnormalities are thought to occur in OCD. In both human and mouse tissues, transcription from the P2 promoter was detected at a lower level compared with the alternatively spliced mRNAs. Thus, all of the alternative EAAC1 transcripts are expressed in a variety of tissues, with similar relative abundance in humans and mice.}

SLC1A1/EAAC1 isoforms inhibit EAAC1-mediated glutamate transport. The transcript derived from the P2 promoter and the two alternatively spliced P1 mRNAs are found in both humans and mice, suggesting that they are evolutionarily conserved and biologically important. When translated, these alternative mRNAs are predicted to give rise to distinct proteins, whereas the transcript from P2 is predicted to give rise to a protein encoded by exons 5-12 (Figure 1d). The lack of exon 2 in ex2skip produces an in-frame deletion of 47 amino acids, which is predicted to eliminate one of the transmembrane helices in the full-length protein (Figure 1d). Similarly, the lack of exon 11 in ex11skip produces another in-frame deletion of 47 amino acids at the carboxyl end (Figure 1d).

To evaluate the biological function of these protein isoforms, we cloned each alternative transcript into a vector containing a carboxyl-terminal FLAG or HA tag to enable protein localization and functional analyses. We first examined glutamate uptake in HEK-293 cells, a cell line that expresses endogenous EAAC1 and can be transfected at almost $100 \%$ efficiency.

The primary EAAC1 transcript, $\mathrm{P} 1$, and all identified alternative isoforms were expressed in HEK-293 cells to determine their action on glutamate uptake (Figure 3a). Glutamate transport conformed to typical saturation kinetics, and plateaued by $60 \mathrm{~min}$ after monitoring activity with ${ }^{3}[\mathrm{H}]$-glutamate (Supplementary Figure 2). Expression of P1 led to significantly higher levels of glutamate uptake compared with nontransfected cells, or cells transfected with an equivalent amount of pcDNA3.1, the vector used to express each EAAC1 isoform (Figure 3a). By contrast, expression of the transcript driven by the second promoter, P2, as well as ex2skip and ex11skip, led to glutamate transport that was significantly lower than that of nontransfected cells (Figure 3a). GTRAP3-18, a protein that binds to EAAC1 and is an established inhibitor of glutamate transport via EAAC1, ${ }^{37}$ also inhibited glutamate transport in HEK-293 cells (Figure 3a). HEK-293 cells normally express endogenous levels of glutamate transporters, including EAAC1. ${ }^{37}$ The inhibition of glutamate uptake did not exceed the inhibition observed when cells were treated with the drug, DL-TBOA, which inhibits active glutamate transport. When isoforms were expressed in HeLa cells, a cell line that lacks EAAC1mediated glutamate uptake, ${ }^{38}$ no active glutamate transport was observed (data not shown). Therefore, the alternative isoforms appear to act as negative regulators of endogenous EAAC1 glutamate uptake.

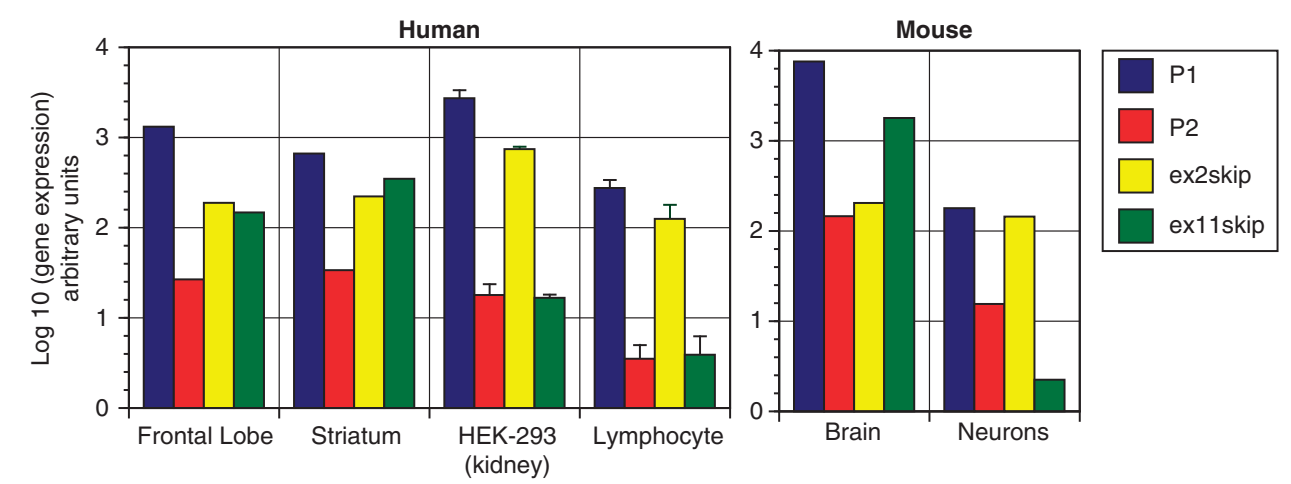

Figure 2 Relative abundance of alternative SLC1A1/EAAC1 transcripts in human and mouse tissues. RNA extracted from the various indicated tissues were subjected to quantitative PCR (see Materials and methods) and normalized to a housekeeping control ( $\beta 2$-microglobulin for humans, $\beta$-actin for mouse). Results are depicted on a logarithmic scale ( \pm s.e.m. where indicated, for HEK-293 cells $(n \geqslant 12)$ or human lymphocytes $(n \geqslant 8)$ ). 
a

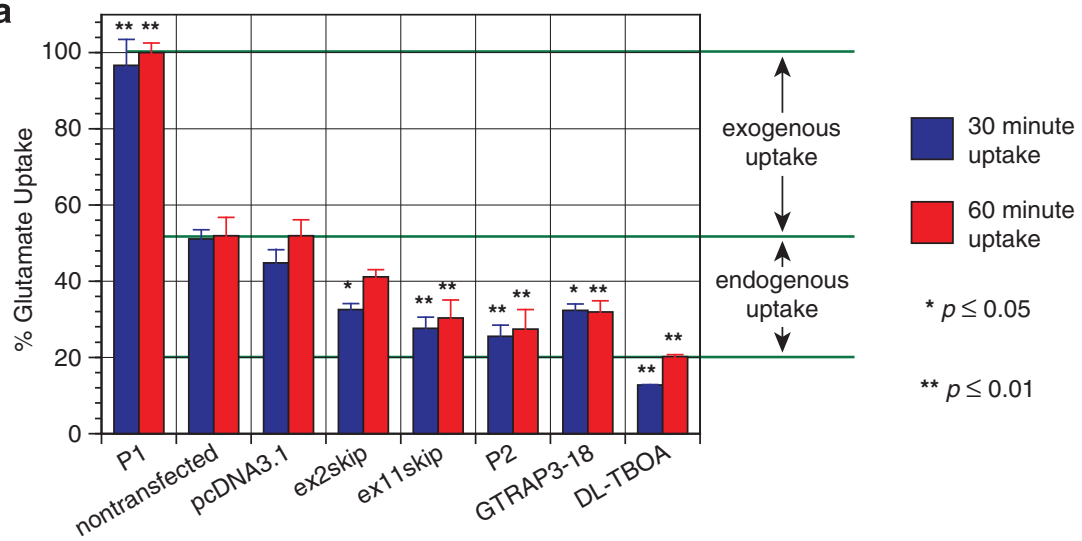

b Other DNA: ex2skip
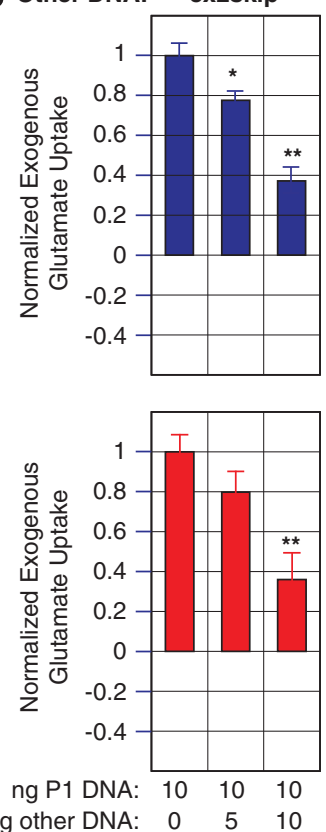

ex11skip
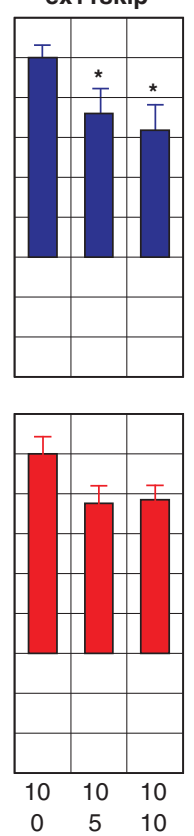

P2
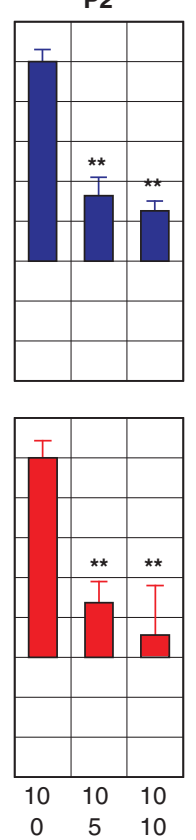

GTRAP3-18
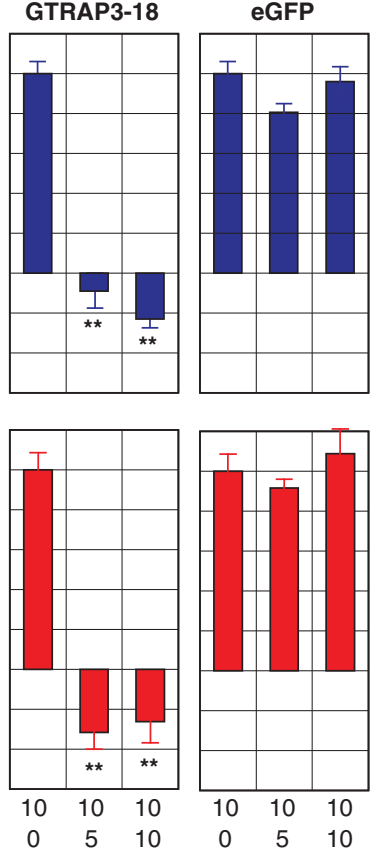

Figure 3 EAAC1 isoforms inhibit glutamate transport from the EAAC1 transporter.(a) EAAC1 isoforms inhibit endogenous glutamate uptake in HEK-293 cells. Normalized glutamate uptake ( 30 or $60 \mathrm{~min}$ ) is depicted in HEK-293 cells transfected with $60 \mathrm{ng}$ of the indicated DNA (the primary transcript of EAAC1 (P1), with each of the EAAC1 isoforms (P2, ex2skip or ex11skip) or GTRAP3-18), non-transfected cells (NT) or cells treated with the drug, DL-TBOA, which inhibits active glutamate transport. Results \pm s.e.m. are shown for triplicate assays conducted in two independent experiments ( $N \geqslant 6)$. ${ }^{*} P \leqslant 0.05$ and ${ }^{* *} P \leqslant 0.01$ in comparison to the pcDNA3.1 control (unpaired two-tailed $t$-test). (b) EAAC1 isoforms inhibit exogenous glutamate uptake derived from the transfected P1 transcript in HEK-293 cells. HEK-293 cells were transfected with $10 \mathrm{ng} \mathrm{P1}$ (in each well of a 96-well plate) and increasing amounts of the indicated other DNA. The total amount of DNA in each well was adjusted to $60 \mathrm{ng}$ using pcDNA3.1. Exogenous glutamate uptake was normalized using $100 \%$ as the maximal uptake observed with $10 \mathrm{ng} \mathrm{P} 1$ and $0 \%$ as the uptake observed with only pcDNA3.1. Results \pm s.e.m. are shown for triplicate assays conducted in two independent experiments $(N \geqslant 6)$ after 30 or 60 min of glutamate uptake. ${ }^{*} P \leqslant 0.05$ and ${ }^{* *} P \leqslant 0.01$ in comparison to maximal uptake in the absence of competitor (unpaired two-tailed $t$-test). eGFP, enhanced green fluorescent protein.

To confirm that the isoforms are indeed acting as negative regulators of glutamate transport via EAAC1, we expressed the primary EAAC1 P1 transcript in HEK-293 cells, and co-expressed increasing amounts of each isoform (Figure 3b). All isoforms significantly inhibited exogenous glutamate transport from the transfected EAAC1 P1 construct (Figure 3b). GTRAP3-18, a control for negative regulation, inhibited both exogenous and endogenous EAAC1 transport, whereas an irrelevant transcript, enhanced green fluorescent protein, had no effect on glutamate transport. Thus, all of the alternative isoforms inhibit glutamate transport via the EAAC1 P1 transcript.
Ex2skip and ex11skip proteins interact with the primary P1 protein. SLC1A1/EAAC1 functions as a trimer. ${ }^{39,40}$ Thus, a possible mechanism by which protein isoforms inhibit glutamate transport is by interfering with the formation of a functional trimeric transporter. To examine this possibility, we first determined if each of the isoforms colocalize with the full-length protein, P1, using HeLa cells, a cell line that lacks expression of endogenous EAAC1 (Figure 4a). ${ }^{38} \mathrm{P} 1$ was tagged with $\mathrm{HA}$, whereas each of the protein isoforms were tagged with FLAG, thus permitting separate visualization of these proteins. ${ }^{32}$ Ex2skip and ex11skip both colocalized with P1, but as expected, P1/P1 colocalization 


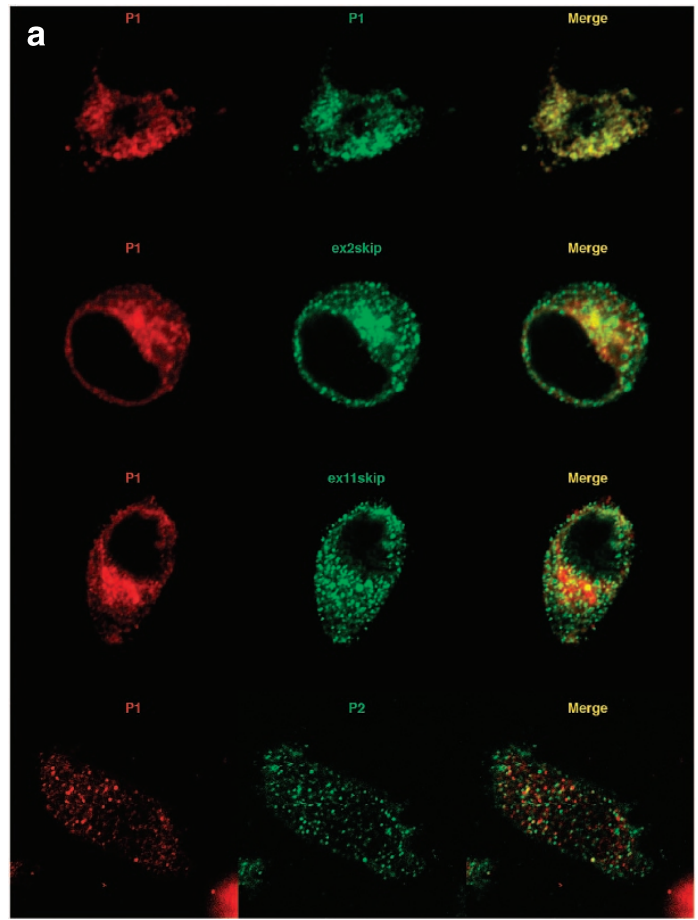

b
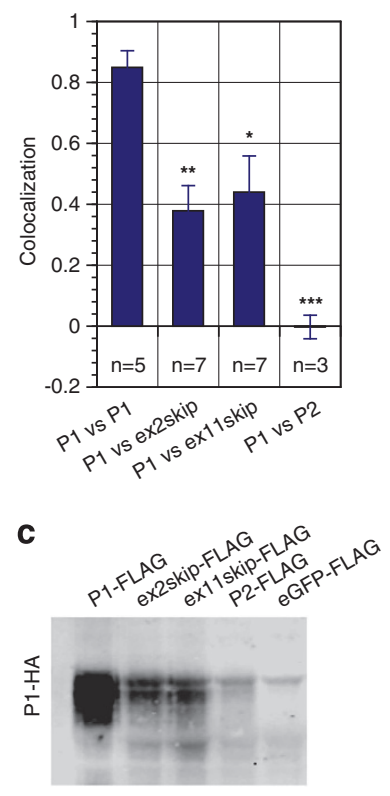

Figure 4 EAAC1 isoforms partially colocalize and interact with the EAAC1 P1 protein in transfected HeLa cells. (a) HeLa cells were transfected with equal amounts of DNA corresponding to P1-HA-tag and to FLAG-tagged isoform (P1, ex2skip, ex11skip, or P2) as indicated. HeLa cells were used in this experiment because they do not express SLC1A1/EAAC1. The hemagglutinin (HA)-tag and FLAG-tag were detected using Alexa555-conjugated anti-rabbit and Alexa647-conjugated anti-mouse antibodies, respectively, and visualized using confocal microscopy as described in Material and methods. Confocal images were optimized for each channel (red $=\mathrm{HA}$; green $=F L A G)$ as indicated. (b) Quantitation ( \pm s.e.m.) of colocalization for FLAG-tag and HA-tag for each of the combinations shown in a, using the method of Costes ${ }^{36}{ }^{*} P \leqslant 0.05,{ }^{* \star} P \leqslant 0.01$ and ${ }^{* * *} P \leqslant 0.001$ in comparison to the P1 vs P1 colocalization. (c) HeLa cells transfected with DNA corresponding to P1-HA-tag and the indicated FLAG-tagged isoform were lysed 2 days post transfection. Lysates were then bound to FLAG-beads, pulled down and proteins resolved by SDS-polyacrylamide gel electrophoresis. HA-tagged P1 was detected using anti-rabbit antibody (see Materials and methods).

was far greater (Figure 4b). By contrast, there was no colocalization observed between the P2 protein isoform and $P 1$. The results indicate that ex2skip and ex11skip proteins partially colocalize to the same cellular regions as the primary $\mathrm{P} 1$ protein, but $\mathrm{P} 2$ protein shows no colocalization with $\mathrm{P} 1$.

To determine if the protein isoforms are indeed physically associated with the primary $\mathrm{P} 1$ protein, transfected HeLa cells were lysed and protein isoforms were pulled down from the lysate using FLAG antibody beads (Figure 4c). The negative control, FLAG-tagged enhanced green fluorescent protein failed to pull down HA-tagged $\mathrm{P} 1$, indicating no interaction between the two proteins. As expected, P1-FLAG avidly pulled down HA-tagged P1. Ex2skip and ex11skip proteins also pulled down HA-tagged P1 but to a lesser extent than P1FLAG. Finally, no physical association was observed between P2-FLAG and HA-tagged P1. These results are consistent with the colocalization data, and support the idea that the ex2skip and ex11skip proteins could inhibit glutamate transport by interfering with proper assembly of the EAAC1 glutamate transporter.

Differential regulation of glutamate signaling in lymphocytes from a clinical population. As analysis of gene expression in living brains is not feasible, and lymphocytes express SLC1A1 as well as a host of genes related to glutamate signaling, they serve as a surrogate model cell system for investigating neural gene regulation. Thus, to determine the relevance of SLC1A1/EAAC1 isoforms in OCD, lymphocytes were isolated from control individuals and patients meeting DSM-IV criteria for OCD. The cells were then cultured in media, in the presence or absence of various reagents, for $24 \mathrm{~h}$ before collection for quantitative gene expression.

As SLC1A1 is expressed in lymphocytes, transcription of this gene was analyzed. We observed that levels of SLC1A1 were decreased by dopamine in lymphocytes from control subjects (Figure 5a). Significantly, subjects with OCD did not exhibit this decrease, suggesting differential regulation of glutamate transporters between controls and OCD. In addition, SLC1A1 expression was significantly higher in OCD compared with controls when lymphocytes were treated with fluoxetine (Figure 5a).

SLC1A1/EAAC1 isoforms showed lower expression in OCD. SLC1A1 isoforms tended to be expressed at a lower level in OCD than controls (Figures $5 b$ and c). Ex11skip displayed significantly lower expression in OCD compared with controls under basal conditions, whereas ex2skip and the P2 transcript displayed significantly lower expression in OCD than controls when lymphocytes were treated with retinoic acid, which enhances the maturation of both lymphocytes and neural cells. ${ }^{41,42}$ As all SLC1A1 isoforms act as negative regulators of SLC1A1, the results suggest 

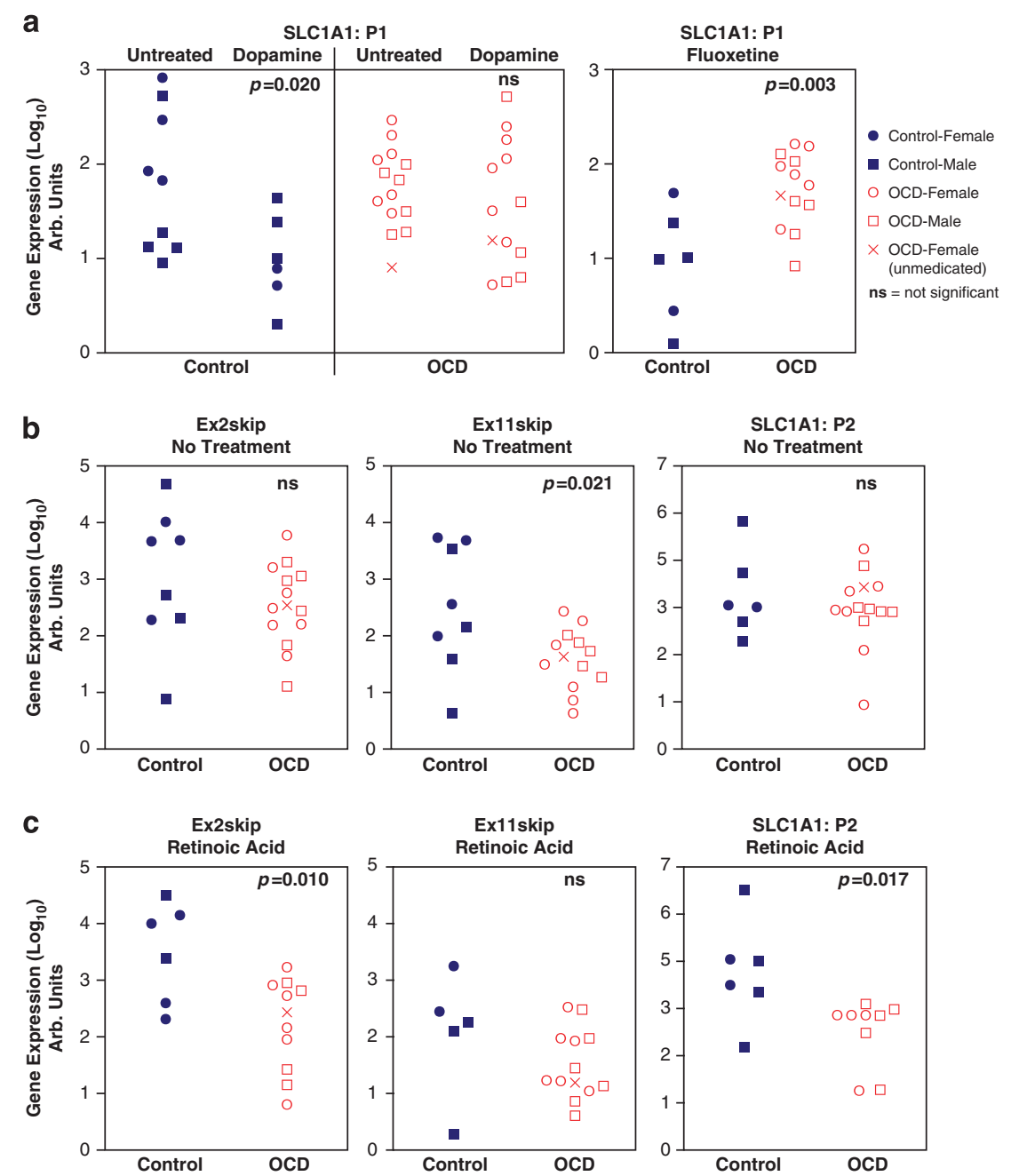

Figure 5 Differential expression of SLC1A1 transcripts in human lymphocytes derived from control subjects and subjects with obsessive-compulsive disorder (OCD). Peripheral lymphocytes were isolated from unrelated controls and individuals with OCD, and cultured with the indicated reagents. All subjects with OCD were treated with serotonin reuptake inhibitors (none were on antipsychotics) except for one drug-naive (unmedicated) female subject with OCD as indicated. Lymphocytes were processed as described in the Materials and methods. Gene expression levels were normalized to $\beta 2$-microglobulin levels, expressed on a logarithmic scale and shown for each individual. (a) Comparative expression of SLC1A1/EAAC1 (P1 transcript) in peripheral lymphocytes under untreated conditions, treated with $1 \mu \mathrm{m}$ dopamine for $24 \mathrm{~h}$ or treated with $10 \mu \mathrm{M}$ fluoxetine for $24 \mathrm{~h}$ in controls and OCD. (b) Basal levels of expression (no treatment) of each SLC1A1 isoform in lymphocytes from the control or OCD group. (c) Comparative expression of each SLC1A1 isoform in lymphocytes treated with $10 \mu \mathrm{m}$ retinoic acid for $24 \mathrm{~h}$.

that under specific developmental conditions, subjects with OCD utilize alternative mechanisms for regulating glutamate transport.

\section{Discussion}

A growing body of evidence from genetic, neuroimaging and clinical studies have pointed to possible dysfunction of the glutamatergic system in the pathogenesis of several major neuropsychiatric disorders, including schizophrenia, ${ }^{43}$ autism spectrum disorder, ${ }^{44}$ and OCD. ${ }^{45-47}$ Perhaps the most compelling genetic finding has been the association of single-nucleotide polymorphisms (SNPs) in the neuronal glutamate transporter gene, SLC1A1/EAAC1, with OCD. In several genetic studies published since 2006, significant associations of SNPS in the SLC1A1/EAAC1 gene were reported in $O C D^{20-25}$ or OCD symptoms in schizophrenia. ${ }^{48,49}$ This is a highly unusual set of findings as multiple replications are extremely rare in psychiatric genetics. In addition, significant association of SNPS in the SLC1A1 gene was reported in schizophrenia ${ }^{50,51}$ and autism, ${ }^{52-54}$ disorders that are comorbid with or share symptom overlap with those of OCD. The SNPs that displayed the most reproducible associations with $O C D$, schizophrenia and autism are located toward the $3^{\prime}$ portion of the EAAC1 gene, and include rs2228622 $23,48,55$ (located in exon 4, near the internal promoter, P2), and rs301979 21,52 and rs301434 ${ }^{20}$ (located near exon 11), and rs $3087879^{24}$ (located in exon12), suggesting the possible involvement of the P2 transcript and/or ex11skip in psychiatric disorders.

SLC1A1/EAAC1 modulates glutamate signaling by transporting glutamate from the extracellular environment to avoid 
excitotoxicity and neuronal death. ${ }^{56}$ However, the genomic regulation of SLC1A1 and possible role in OCD is unclear. Here, we describe three isoforms, all of which act as negative modulators of SLC1A1/EAAC1 function. As expected, given the deletions in transmembrane regions, all three isoforms lack inherent glutamate transport function. The two alternatively spliced protein isoforms (ex2skip and ex11skip) interact with and partially colocalize with the primary EAAC1 P1 protein, suggesting they impair assembly of a functional trimeric transporter. ${ }^{39}$ The third isoform (P2), generated from a second internal promoter, does not interact with the EAAC1 P1 protein, suggesting that it may act at the RNA level by interfering with translation of the $\mathrm{P} 1$ protein. The protein, GTRAP3-18, is a potent negative modulator of SLC1A1/ EAAC1. ${ }^{37}$ The existence of several independently operating modulators of SLC1A1/EAAC1 function adds an unexpected layer of complexity to the regulation of this transporter.

All of the alternative isoforms are evolutionarily conserved between humans and mice, and were found in brain, lymphocytes and kidney cells under nonpathological conditions. There is a relatively high abundance of the alternatively spliced EAAC1 mRNAs in striatum, a brain region involved in OCD. ${ }^{57}$ Accordingly, these isoforms are likely to act as natural regulators of EAAC1 function.

Given the strong evidence implicating glutamate dysregulation in OCD, we sought a biological system to study the expression of SLC1A1. Previous research has shown that SNPs in the vicinity of the SLC1A1 gene, including those strongly associated with OCD, predict the expression of SLC1A1 in both brain and lymphoblastoid cells. ${ }^{24}$ This study suggests that genetic polymorphisms are strong determinants of the expression and regulation of SLC1A1 in brain as well as lymphoblastoid cells, which are immortalized lymphocytes. Accordingly, we used human lymphocytes as a peripheral system to analyze the regulation of SLC1A1 isoforms. Peripheral lymphocytes are easily accessible and express all SLC1A1 isoforms described here, including other neural genes relevant to glutamate biology. A limitation of this study, however, is that almost all the subjects with OCD have been treated with medications.

Our initial results suggest that the regulation of glutamate transporters in lymphocytes resembles that observed in the brain. Levels of SLC1A1 were decreased by treatment with dopamine in lymphocytes from control subjects, a scenario similar to the downregulation of high-affinity glutamate transport by dopamine in the striatum, a normal physiological response. ${ }^{58}$

To determine if there are biological differences in OCD, we asked whether expression of SLC1A1 is differentially regulated in OCD compared with controls. In contrast to controls, there was no significant downregulation of SLC1A1 in dopamine-treated lymphocytes from OCD subjects. Moreover, SLC1A1 transcription was increased in response to fluoxetine in subjects with OCD but showed no change in controls. This is relevant as selective serotonin reuptake inhibitors such as fluoxetine are the main treatment for OCD. Thus, differential regulation of SLC1A1 was observed between OCD and controls.

In addition, differential expression of all SLC1A1 isoforms was observed between OCD and controls. Basal levels of ex11skip were significantly lower in lymphocytes derived from OCD subjects compared with controls. In lymphocytes treated with retinoic acid, expression of all SLC1A1 alternative isoforms was significantly decreased in OCD compared with controls. Thus, all SLC1A1 isoforms show differential regulation in lymphocytes derived from OCD compared with controls.

The differential regulation of the SLC1A1 primary transcript and its isoforms in OCD raises new possibilities regarding the role of glutamate in this disorder. As the isoforms act as negative modulators of SLC1A1 function, lower levels in OCD could represent a mechanism to compensate for increased local concentrations of extracellular glutamate. This notion would fit with prior observations reporting increased glutamate in the cerebrospinal fluid of patients with OCD, $, 7,7$ imaging studies showing increased levels of glutamate in the caudate nucleus and orbitofrontal cortex, ${ }^{3,4}$ and increased excitability in the brains of individuals with OCD. ${ }^{59,60}$ However, glutamate levels have also been reported to be decreased in the caudate nucleus of pediatric cases of OCD, ${ }^{1}$ and decreased in the anterior cingulate gyrus of patients with OCD., ${ }^{2,5}$ Indeed, the notion that only hyperglutamatergic activity underlies OCD symptoms may be too simplistic, as glutamate regulation in the brain is complex. ${ }^{45}$ Moreover, glutamatergic neurons are not found in the striatum, ${ }^{61}$ suggesting that there may be alternative functions for this neurotransmitter in this region. ${ }^{45}$ Our finding that SLC1A1 is regulated by a suite of negatively acting modulators, in addition to GTRAP3 $-18,{ }^{37}$ is consistent with the complexity of glutamate regulation.

SLC1A1 is also differentially regulated by dopamine and fluoxetine in OCD compared with controls. Whether these are medication-related effects or not will require future studies. Moreover, if glutamate is the primary neurotransmitter involved in OCD, this observation raises the question as to whether the therapeutic effects of medication (that is, selective serotonin re-uptake inhibitors or dopamine antagonists) are in part due to their effects on the regulation of SLC1A1.

To confirm the lymphocyte studies, experiments should be replicated in a larger clinical population, assay conditions optimized and medication effects controlled. Studies in a pediatric population would be informative, as pediatric OCD is more familial, ${ }^{62}$ and the potentially confounding effects of illness duration and treatment intervention may be minimized. ${ }^{63}$ Future studies will also focus on establishing the role of genetic polymorphisms on the regulation of SLC1A1.

\section{Conflict of interest}

The authors declare no conflict of interest.

Acknowledgements. We thank Albert Lin for technical assistance. This research was supported by internal funds from Butler Hospital and partly conducted at the Genomics Core Facility at Brown University (partial support from NIGMS P30GM103410, NCRR (P30RR031153, P20RR018728 and S10RR02763), and NSF (EPSCoR 0554548)) and also supported by the National Alliance for Research on Schizophrenia and Depression (NARSAD).

1. Rosenberg DR, MacMaster FP, Keshavan MS, Fitzgerald KD, Stewart CM, Moore GJ. Decrease in caudate glutamatergic concentrations in pediatric obsessive-compulsive 
disorder patients taking paroxetine. J Am Acad Child Adolesc Psychiatry 2000; 39 : 1096-1103.

2. Rosenberg DR, Mirza Y, Russell A, Tang J, Smith JM, Banerjee SP et al. Reduced anterior cingulate glutamatergic concentrations in childhood $O C D$ and major depression versus healthy controls. J Am Acad Child Adolesc Psychiatry 2004; 43: 1146-1153.

3. Starck G, Ljungberg M, Nilsson M, Jonsson L, Lundberg S, Ivarsson T et al. A $1 \mathrm{H}$ magnetic resonance spectroscopy study in adults with obsessive compulsive disorder: relationship between metabolite concentrations and symptom severity. J Neural Transm 2008; 115 1051-1062.

4. Whiteside SP, Port JD, Deacon BJ, Abramowitz JS. A magnetic resonance spectroscopy investigation of obsessive-compulsive disorder and anxiety. Psychiatry Res 2006; 146 137-147.

5. Yucel M, Wood SJ, Wellard RM, Harrison BJ, Fornito A, Pujol J et al. Anterior cingulate glutamate-glutamine levels predict symptom severity in women with obsessive-compulsive disorder. Aust N Z J Psychiatry 2008; 42: 467-477.

6. Chakrabarty K, Bhattacharyya S, Christopher R, Khanna S. Glutamatergic dysfunction in OCD. Neuropsychopharmacology 2005; 30: 1735-1740.

7. Bhattacharyya S, Khanna S, Chakrabarty K, Mahadevan A, Christopher R, Shankar SK Anti-brain autoantibodies and altered excitatory neurotransmitters in obsessive-compulsive disorder. Neuropsychopharmacology 2009; 34: 2489-2496.

8. Nordstrom EJ, Burton FH. A transgenic model of comorbid Tourette's syndrome and obsessive-compulsive disorder circuitry. Mol Psychiatry 2002; 7: 617-625; 524.

9. Welch JM, Lu J, Rodriguiz RM, Trotta NC, Peca J, Ding JD et al. Cortico-striatal synaptic defects and OCD-like behaviours in Sapap3-mutant mice. Nature 2007; 448: 894-900.

10. Shmelkov SV, Hormigo A, Jing D, Proenca CC, Bath KG, Milde T et al. Slitrk5 deficiency impairs corticostriatal circuitry and leads to obsessive-compulsive-like behaviors in mice. Nat Med 2010; 16: 598-602; 591p following 602.

11. Coric V, Taskiran S, Pittenger C, Wasylink S, Mathalon DH, Valentine $\mathrm{G}$ et al. Riluzole augmentation in treatment-resistant obsessive-compulsive disorder: an open-label trial. Biol Psychiatry 2005; 58: 424-428.

12. Grant $P$, Lougee L, Hirschtritt M, Swedo SE. An open-label trial of riluzole, a glutamate antagonist, in children with treatment-resistant obsessive-compulsive disorder. J Child Adolesc Psychopharmacol 2007; 17: 761-767.

13. Pittenger C, Kelmendi B, Wasylink S, Bloch MH, Coric V. Riluzole augmentation in treatment-refractory obsessive-compulsive disorder: a series of 13 cases, with long-term follow-up. J Clin Psychopharmacol 2008; 28: 363-367.

14. Poyurovsky M, Weizman R, Weizman A, Koran L. Memantine for treatment-resistant OCD. Am J Psychiatry 2005; 162: 2191-2192.

15. Pasquini M, Biondi M. Memantine augmentation for refractory obsessive-compulsive disorder. Prog Neuropsychopharmacol Biol Psychiatry 2006; 30: 1173-1175.

16. Aboujaoude E, Barry JJ, Gamel N. Memantine augmentation in treatment-resistant obsessive-compulsive disorder: an open-label trial. J Clin Psychopharmacol 2009; 29: 51-55.

17. Feusner JD, Kerwin L, Saxena S, Bystritsky A. Differential efficacy of memantine for obsessive-compulsive disorder vs generalized anxiety disorder: an open-label trial. Psychopharmacol Bull 2009; 42: 81-93.

18. Stewart SE, Jenike EA, Hezel DM, Stack DE, Dodman NH, Shuster L et al. A single-blinded case-control study of memantine in severe obsessive-compulsive disorder. J Clin Psychopharmacol 2010; 30: 34-39.

19. Lafleur DL, Pittenger C, Kelmendi B, Gardner T, Wasylink S, Malison RT et al $\mathrm{N}$-Acetylcysteine augmentation in serotonin reuptake inhibitor refractory obsessivecompulsive disorder. Psychopharmacology (Berl) 2006; 184: 254-256.

20. Arnold PD, Sicard T, Burroughs E, Richter MA, Kennedy JL. Glutamate transporter gene SLC1A1 associated with obsessive-compulsive disorder. Arch Gen Psychiatry 2006; 63: 769-776.

21. Dickel DE, Veenstra-VanderWeele J, Cox NJ, Wu X, Fischer DJ, Van Etten-Lee M et al. Association testing of the positional and functional candidate gene SLC1A1/ EAAC1 in early-onset obsessive-compulsive disorder. Arch Gen Psychiatry 2006; 63 : 778-785.

22. Shugart YY, Wang Y, Samuels JF, Grados MA, Greenberg BD, Knowles JA et al. A familybased association study of the glutamate transporter gene SLC1A1 in obsessivecompulsive disorder in 378 families. Am J Med Genet B Neuropsychiatr Genet 2009; 150B: 886-892.

23. Stewart SE, Fagerness JA, Platko J, Smoller JW, Scharf JM, Illmann C et al. Association of the SLC1A1 glutamate transporter gene and obsessive-compulsive disorder. Am J Med Genet B Neuropsychiatr Genet 2007; 144B: 1027-1033.

24. Wendland JR, Moya PR, Timpano KR, Anavitarte AP, Kruse MR, Wheaton MG et al. A haplotype containing quantitative trait loci for SLC1A1 gene expression and its association with obsessive-compulsive disorder. Arch Gen Psychiatry 2009; 66: 408-416.

25. Samuels J, Wang Y, Riddle MA, Greenberg BD, Fyer AJ, McCracken JT et al Comprehensive family-based association study of the glutamate transporter gene SLC1A1 in obsessive-compulsive disorder. Am J Med Genet B Neuropsychiatr Genet 2011; 156B: 472-477.

26. Hanna GL, Veenstra-VanderWeele J, Cox NJ, Boehnke M, Himle JA, Curtis GC et al. Genome-wide linkage analysis of families with obsessive-compulsive disorder ascertained through pediatric probands. Am J Med Genet 2002; 114: 541-552.
27. Willour VL, Yao Shugart Y, Samuels J, Grados M, Cullen B, Bienvenu OJ III et al. Replication study supports evidence for linkage to 9p24 in obsessive-compulsive disorder. Am J Hum Genet 2004; 75: 508-513.

28. Takai D, Jones PA. The CpG island searcher: a new WWW resource. In Silico Bio/ 2003; 3 : 235-240.

29. Jin XP, Peng JB, Huang F, Zhu YN, Fei J, Guo LHA. mRNA molecule encoding truncated excitatory amino acid carrier 1 (EAAC1) protein (EAAC2) is transcribed from an independent promoter but not an alternative splicing event. Cell Res 2002; 12: 257-262.

30. Matsumoto $\mathrm{Y}$, Enomoto $\mathrm{T}$, Masuko $\mathrm{T}$. Identification of truncated human glutamate transporter. Tohoku J Exp Med 1999; 187: 173-182.

31. Sambrook J, Fritsch EF, Maniatis T. Molecular Cloning. A Laboratory Manual, 2nd edn. Cold Spring Harbor Laboratory Press: Cold Spring Harbor, 1989.

32. Malhotra A. Tagging for protein expression. Methods Enzymol 2009; 463: 239-258.

33. Applied_Biosystems User Bulletin \#2, ABI PRISM 7700 Sequence detection system. P/N 4303859B. Applied Biosystems, Inc: Foster City, CA, 2001.

34. Schefe JH, Lehmann KE, Buschmann IR, Unger T, Funke-Kaiser H. Quantitative real-time RT-PCR data analysis: current concepts and the novel 'gene expression's CT difference' formula. J Mol Med 2006; 84: 901-910.

35. Porton B, Ferreira A, DeLisi LE, Kao HT. A rare polymorphism affects a mitogen-activated protein kinase site in synapsin III: possible relationship to schizophrenia. Biol Psychiatry 2004; 55: 118-125.

36. Costes SV, Daelemans D, Cho EH, Dobbin Z, Pavlakis G, Lockett S. Automatic and quantitative measurement of protein-protein colocalization in live cells. Biophys $\mathrm{J} 2004 ; 86$ : 3993-4003.

37. Lin Cl, Orlov I, Ruggiero AM, Dykes-Hoberg M, Lee A, Jackson M et al. Modulation of the neuronal glutamate transporter EAAC1 by the interacting protein GTRAP3-18. Nature 2001; 410: 84-88.

38. Bjoras M, Gjesdal O, Erickson JD, Torp R, Levy LM, Ottersen OP et al. Cloning and expression of a neuronal rat brain glutamate transporter. Brain Res Mol Brain Res 1996; 36: 163-168.

39. Yernool D, Boudker O, Jin Y, Gouaux E. Structure of a glutamate transporter homologue from Pyrococcus horikoshii. Nature 2004; 431: 811-818.

40. Grewer C, Balani P, Weidenfeller C, Bartusel T, Tao Z, Rauen T. Individual subunits of the glutamate transporter EAAC1 homotrimer function independently of each other. Biochemistry 2005; 44: 11913-11923.

41. Hall JA, Grainger JR, Spencer SP, Belkaid $Y$. The role of retinoic acid in tolerance and immunity. Immunity 2011; 35: 13-22.

42. Maden M. Retinoic acid in the development, regeneration and maintenance of the nervous system. Nat Rev Neurosci 2007; 8: 755-765.

43. Kantrowitz J, Javitt DC. Glutamatergic transmission in schizophrenia: from basic research to clinical practice. Curr Opin Psychiatry 2012; 25: 96-102.

44. Carlson GC. Glutamate receptor dysfunction and drug targets across models of autism spectrum disorders. Pharmacol Biochem Behav 2012; 100: 850-854.

45. Pittenger $\mathrm{C}$, Bloch MH, Williams K. Glutamate abnormalities in obsessive compulsive disorder: neurobiology, pathophysiology, and treatment. Pharmacol Ther 2011; 132 : 314-332.

46. Wu K, Hanna GL, Rosenberg DR, Arnold PD. The role of glutamate signaling in the pathogenesis and treatment of obsessive-compulsive disorder. Pharmacol Biochem Behav 2012; 100: 726-735.

47. Nestadt G, Grados M, Samuels JF. Genetics of obsessive-compulsive disorder. Psychiat Clin North Am 2010; 33: 141-158.

48. Kwon JS, Joo YH, Nam HJ, Lim M, Cho EY, Jung MH et al. Association of the glutamate transporter gene SLC1A1 with atypical antipsychotics-induced obsessive-compulsive symptoms. Arch Gen Psychiatry 2009; 66: 1233-1241.

49. Schirmbeck F, Nieratschker V, Frank J, Englisch S, Rausch F, Meyer-Lindenberg A et al. Polymorphisms in the glutamate transporter gene SLC1A1 and obsessive-compulsive symptoms induced by second-generation antipsychotic agents. Psychiatr Genet 2012; 22: 245-252.

50. Deng X, Shibata H, Takeuchi N, Rachi S, Sakai M, Ninomiya H et al. Association study of polymorphisms in the glutamate transporter genes SLC1A1, SLC1A3, and SLC1A6 with schizophrenia. Am J Med Genet B Neuropsychiatr Genet 2007; 144B: 271-278.

51. Horiuchi $Y$, lida S, Koga M, Ishiguro H, lijima Y, Inada T et al. Association of SNPs linked to increased expression of SLC1A1 with schizophrenia. Am J Med Genet B Neuropsychiatr Genet 2012; 159B: 30-37.

52. Brune CW, Kim SJ, Hanna GL, Courchesne E, Lord C, Leventhal BL et al. Family-based association testing of OCD-associated SNPs of SLC1A1 in an autism sample. Autism Res 2008; 1: 108-113.

53. Gadow KD, Roohi J, DeVincent CJ, Kirsch S, Hatchwell E. Glutamate transporter gene (SLC1A1) single nucleotide polymorphism (rs301430) and repetitive behaviors and anxiety in children with autism spectrum disorder. J Autism Dev Disord 2010; 40: 1139-1145.

54. Kantojarvi K, Onkamo P, Vanhala R, Alen R, Hedman M, Sajantila A et al. Analysis of 9p24 and 11p12-13 regions in autism spectrum disorders: rs1340513 in the JMJD2C gene is associated with ASDs in Finnish sample. Psychiatr Genet 2010; 20: 102-108.

55. Ryu S, Oh S, Cho EY, Nam HJ, Yoo JH, Park T et al. Interaction between genetic variants of DLGAP3 and SLC1A1 affecting the risk of atypical antipsychotics-induced obsessive-compulsive symptoms. Am J Med Genet B Neuropsychiatr Genet 2011; 156B: 949-959. 
56. Tzingounis AV, Wadiche Jl. Glutamate transporters: confining runaway excitation by shaping synaptic transmission. Nat Rev Neurosci 2007; 8: 935-947.

57. Insel TR, Winslow JT. Neurobiology of obsessive compulsive disorder. Psychiatr Clin North Am 1992; 15: 813-824.

58. Kerkerian L, Dusticier N, Nieoullon A. Modulatory effect of dopamine on high-affinity glutamate uptake in the rat striatum. J Neurochem 1987; 48: 1301-1306.

59. Greenberg BD, Ziemann U, Cora-Locatelli G, Harmon A, Murphy DL, Keel JC et al. Altered cortical excitability in obsessive-compulsive disorder. Neurology 2000; 54: 142-147.

60. Greenberg BD, Ziemann U, Harmon A, Murphy DL, Wassermann EM. Decreased neuronal inhibition in cerebral cortex in obsessive-compulsive disorder on transcranial magnetic stimulation. Lancet 1998; 352: 881-882.

61. Kreitzer AC. Physiology and pharmacology of striatal neurons. Annu Rev Neurosci 2009; 32: 127-147.
62. Grados M, Wilcox HC. Genetics of obsessive-compulsive disorder: a research update. Expert Rev Neurotherapeutics 2007; 7: 967-980.

63. Szeszko PR, MacMillan S, McMeniman M, Chen S, Baribault K, Lim KO et al. Brain structural abnormalities in psychotropic drug-naive pediatric patients with obsessive-compulsive disorder. Am J Psychiatry 2004; 161: 1049-1056.

cc)(i)(-) Translational Psychiatry is an open-access journal published by Nature Publishing Group. This work is licensed under a Creative Commons Attribution-NonCommercialShareAlike 3.0 Unported License. To view a copy of this license, visit http://creativecommons.org/licenses/by-nc-sa/3.0/

Supplementary Information accompanies the paper on the Translational Psychiatry website (http://www.nature.com/tp) 